\title{
Improving the IRIS Recognition Under Different Illumination Using RETINEX Algorithms
}

\author{
Shruthi A. Kumar ${ }^{1 *}$, A. Baskar ${ }^{2}$ \\ ${ }^{I}$ Department Of Computer Science And Engineering, Amrita School Of Engineering, Coimbatore. \\ Amrita Vishwa Vidyapeetham, India. \\ ${ }^{2}$ Department Of Computer Science And Engineering, Amrita School Of Engineering, Coimbatore. \\ Amrita Vishwa Vidyapeetham, India. \\ E-Mail:A_Baskar@Cb.Amrita.Edu \\ *Corresponding Author E-Mail:Cb.En.P2cvi16007@Cb.Students.Amrita.Edu
}

\begin{abstract}
Iris detection and recognition provides more accurate and secure authentication nowadays. The probability of any two people having the same iris pattern is nearly zero, even the identical twins will not have the same iris pattern. The noise and illumination changes, challenges iris recognition correctness and security in authentication process. The available recent pre-processing techniques for iris detection address different type of noise suppression and removing unwanted information in iris, but still it strives with illumination issues. In this paper, we proposed Retinex algorithm for improving iris detection rate. The proposed work comprises into three steps: First we proposed Retinex algorithm in pre-processing, it works based on reflectance value of image and skips the illumination value in image, subsequently feature extraction uses Gabor filter for iris code generation. In conclusion, distance metrics Hamming distance used for iris recognition the proposed work evaluated MMU iris database under different illumination conditions and provides better results.
\end{abstract}

Keywords: Single Scale RETINEX, multi scale RETINEX, IRIS enhancement, GABOR filter.

\section{Introduction}

Iris Recognition is one the most secured means of biometrics technology because of its uniqueness. It contains a lot of wrinkles, stripes, spots etc. which makes it unique. It's difficult to manipulate the iris recognition system. Iris recognition (2) involves 3 major steps: pre-processing, feature extraction (3), recognition. Pre-processing involves the enhancement of the image data obtained i.e. removing the noise, edge detection, localising the iris region, normalization and segmentation of iris (4). Feature extraction includes the extracting the features of the iris. Recognition involves comparing the feature obtained with the feature in the database to check whether it matches or not (5).

The major problem of the existing system is that, it fails to address the illumination problem. It's not illumination invariant. The results will get affected if illumination varies. It will reduce the recognition rate of the system. Retinex Theory tries to reduce the difference between the original scene and the image captured. It consists of estimation the normalization of illumination. In general, Retinex algorithm works based on reflectance value of image and skips the illumination value in image. Based on Retinex theory(1), Single Scale Retinex (SSR) is the basic algorithm, which provides either Dynamic Range Compression (DRC) or tonal analysis but not simultaneously. The Multi Scale Retinex (MSR) can provide both. So Retinex algorithm enhances the image which has illumination deficiencies.

In this paper, we proposed Retinex algorithm for improving iris detection rate. The proposed work comprises into three steps: First we proposed Retinex algorithm in pre-processing, it works based on reflectance value of image and skips the illumination value in image, subsequently feature extraction uses Gabor filter for iris code generation. In conclusion, distance metrics Hamming distance used for iris recognition.

\section{Proposed Work}

The proposed work comprises into three steps: First we proposed Retinex algorithm in pre-processing, it works based on reflectance value of image and skips the illumination value in image, subsequently feature extraction uses Gabor filter for iris code generation. In conclusion, distance metrics Hamming distance uses for iris recognition.

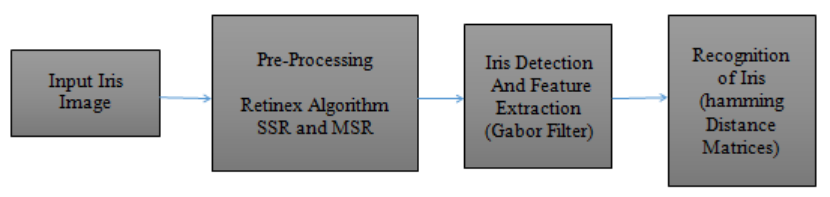

Fig 1: Block diagram of proposed work

\section{Pre-processing}

Retinex Theory: It improves the image quality of an image when the lighting of an image is not good. Humans can see the colors correctly even in low light, are not the case for the devices. The Retinex theory benefits to reduce the gap between the human vision and the machine vision. In general, image is considered as the product of reflectance and illumination. It illustrates in the equation 1 .

$S(x, y)=R(x, y) * L(x, y)$

Where, 
$S$ is the observed image value ranging from 0 to 255 ,

$R$ is the reflectance value lies in $[0,1]$

$L$ is illumination ranging from $[0,255]$.

The Retinex theory uses assumptions called the illumination vary smoothly and it skips illumination and estimates reflectance values. $\mathrm{R}$ should be between 0 and 1 and $\mathrm{L} \geq S$, the reflectance part contains the higher frequency information like the edge, texture. The Retinex algorithm converts the image to logarithmic domain, it describes in the equation 2 .

$$
\log S=\log R+\log L
$$

Single Scale Retinex (SSR):It consists of a logarithmic illumination detector function that estimates the vision system and is based on the centre function. The centre function is alike to the Difference of Gaussian (DoG) function. SSR is given by equation 3.SSR cannot provide DRC and tonal analysis simultaneously. It produces halos about the objects. It gives the normalized reflectance $\mathrm{R}$ and luminance $\mathrm{L}$ as the output.

$R_{i}(x, y)=\log _{i}(x, y) \log \left[F(x, y) * I_{i}(x, y)\right]$

Where,

$I_{i}(x, y)$ - The image in RGB

$R_{i}(x, y)$ - The Retinex output image

$F(x, y)$ - Gaussian function.

Multi Scale Retinex (MSR): The DRC and getting tonal analysis simultaneously, MSR is introduced. The processing speed is improved since the processing of different sampled versions is done parallel. The equation 4 illustrates this. For MSR, the values of scale, number of scale, weights are important. Generally, 15, 80, 250 are the fixed scales were used. To obtain the major objective of MSR that is to obtain the compression of dynamic range and getting colour rendition, for those weights should be selected with utmost care.

$$
\begin{gathered}
R_{M S R i}=\sum_{n=1}^{N} w_{n}\left\{\log _{i}(x, y) \log [F(x, y)\right. \\
\left.\left.* I_{i}(x, y)\right]\right\}
\end{gathered}
$$

Where,

$\mathrm{N}$ is the number of scales

$w$ is the weight of the scale.

Iris Detection: The first step will be locating the pupil edge and can be done accurately since the pupil boundary is circular. The method to find pupil should be robust to the extreme conditions like higher brightness of pupil than the iris because of the reflections from cornea. The optimization is used for pupil boundary detection. The parameter space is examined for the best values for centre co-ordinates of the circle $\left(x_{0}, y_{0}\right)$ and the radius $r$. To find these three parameters, integro- differential operator (1) can be used. The equation 5 describes this.

$\max _{\left(r, x_{0}, y_{0}\right)}\left|G_{\sigma}(r) * \frac{\partial}{\partial \mathrm{r}} \oint_{\left(r, x_{0}, y_{0}\right)} \frac{I(x, y)}{2 \pi r} d s\right|$

Here the $I(x, y)$ is the image. The * operation denotes convolution and $G_{\sigma}$ is the Gaussian kernel of scale $\sigma$. The integro- differential operator works like a circular edge detector through the parameter space defined by circle center coordinates $\left(x_{0}, y_{0}\right)$ and the radius $\mathrm{r}$. This operator detects the inner pupil boundary and the outer limbic boundary of the iris. Daugman's rubber sheet model: used for normalizing the segmented iris feature. It converts circular shape into rectangular rubber sheet; correspondingly it converted to polar coordinates.

\section{Feature extraction and recognition}

1D Log-Gabor filter: used for the extraction of the features. Features are determined by convolving the normalized iris image with the 1D Log-Gabor filter (6) and is phase quantised to generate a bitwise biometric template. Hamming distance can be used to find the distance between reference image template and the input image template. Threshold value should be set first to determine whether the both images are matching or not. If the distance is more than the threshold, iris is not matching, else it is matching.

\section{Results and Analysis}

The proposed work implements Retinex algorithm for improving iris detection and recognition rate. We tested algorithm using MMU iris database. The results were analyzed into three different cases: 1. Original MMU database Images. 2. The MMU database images altered with reducing contrast by $40 \%$. 3. The MMU database images altered with increasing contrast and brightness by $40 \%$.

Database: The standard dataset of Multimedia University (MMU) is used for the work. It consists of 450 images, 5 images per iris and 2 irises per subject. All images were captured using LG Iris Access 2200 at a range of 7-25 centimeters.

CASE1: Original MMU Iris Database Images: This case we used original MMU database without modifying. System was able to detect the iris region correctly for both Retinex algorithms and the traditional pre-processing techniques (histogram equalization, Gaussian filter). The figure 2.a shows original MMU dataset sample image, 2.b shows the traditional pre-processing approach, 2.cshows SSR based output and 2.d shows MSR based output
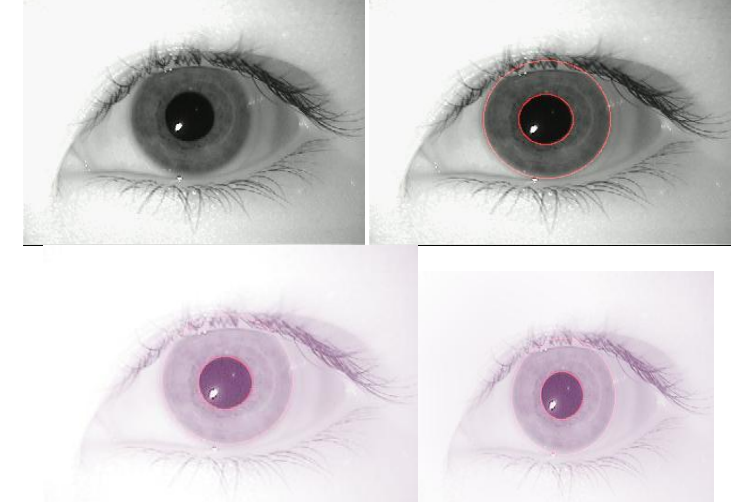

Fig 2: (a)original MMU dataset sample image, (b) The traditional pre-processing approach, (c) SSR based output; (d) MSR based output

CASE2:The MMU database images altered with contrast by 40\%:The original MMU database altered with reducing $40 \%$ of contrast. The result shows traditional approach fails in detection and SSR, MSR properly detect the iris region. The figure 3.a shows contrast reduced MMU dataset sample image, 3.b shows the traditional pre-processing approach, 3.c SSR based output and 3.d shows MSR based output.

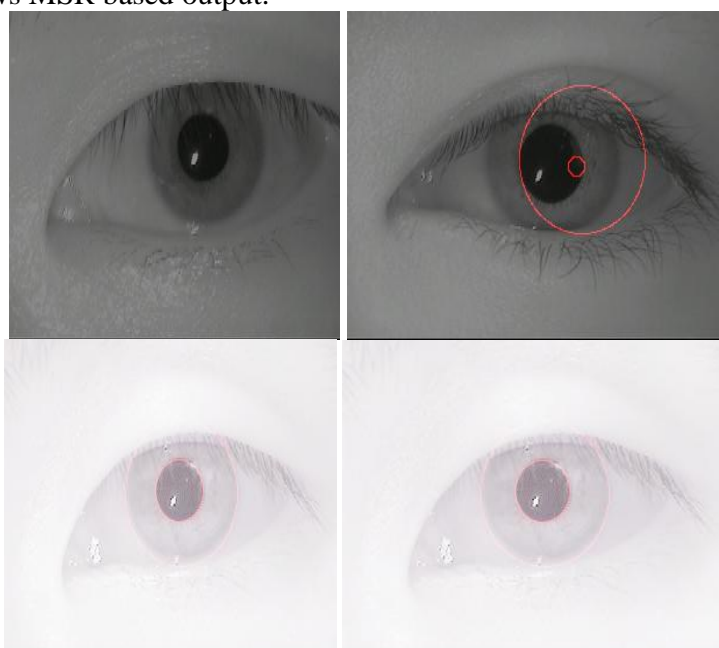

Fig 3: (a) contrast reduced MMU dataset sample image, (b) output of traditional approach (c) SSR based output, (d) MSR based output.

CASE3: The MMU database images altered with contrast and brightness by $\mathbf{4 0 \%}: 40 \%$ of brightness is reduced, $40 \%$ of contrast 
is increased to create the next set of data for the Iris Recognition System.

The result shows traditional approach fails in pupil detection and SSR, MSR properly detect the pupil region. The figure 4.a shows contrast increased MMU dataset sample image, 4.b shows the traditional pre-processing approach, 4.c SSR based output and 4.d shows MSR based output.

The figure 5 illustrates SSR and MSR fails in iris detection for very high contrast changes.

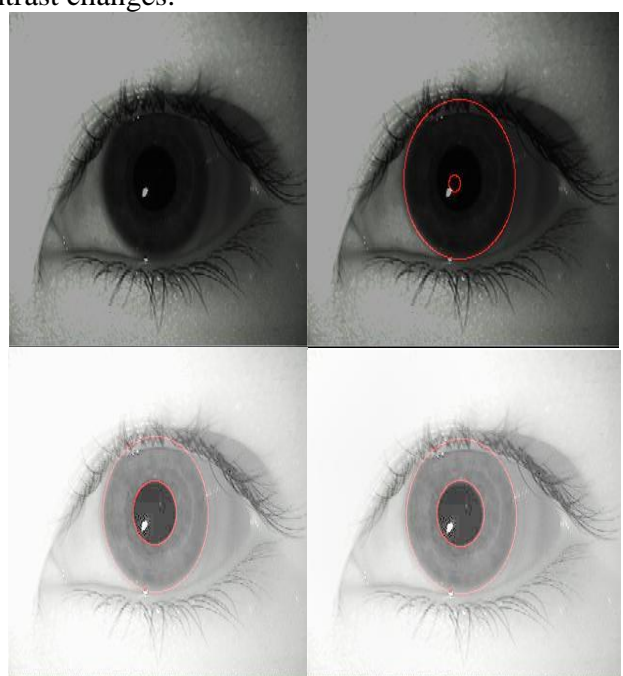

Fig 4: (a) contrast increased MMU dataset sample image, (b) output of traditional approach (c) SSR based output, (d) MSR based output.

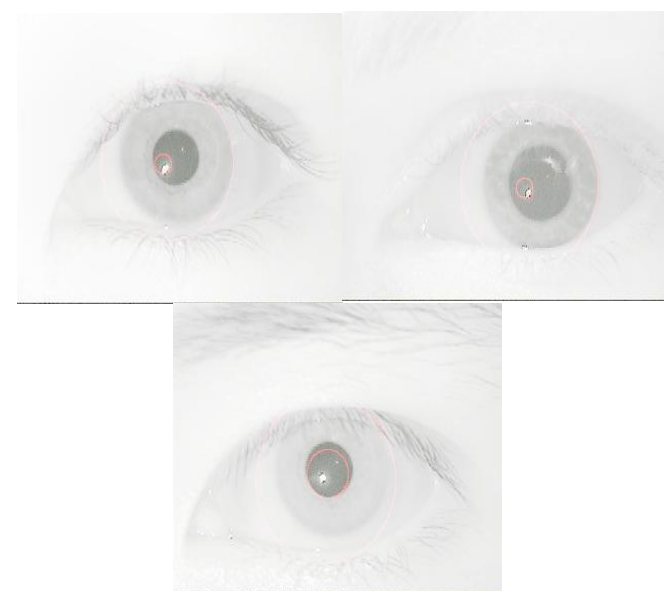

Fig 5: Describes SSR and MSR results, it fails in iris detection for very high contrast changes.

The table1 describes performance analysis of Retinex algorithm vs. traditional pre-processing techniques. Table shows SSR and MSR performs better compare to traditional pre-processing techniques. The analysis shows SSR and MSR fails in iris detection for very high contrast changes. Subsequently, the figure 6 illustrates performance analysis of Retinex algorithm vs. traditional pre-processing techniques.

Table 1: Performance Analysis of Retinex Algorithm vs. Traditional

\begin{tabular}{|c|c|c|c|c|}
\hline \multicolumn{1}{|c|}{ Pre-Processing Techniques } & $\begin{array}{c}\text { No of } \\
\text { Input } \\
\text { Images }\end{array}$ & SSR & MSR & $\begin{array}{c}\text { Traditional } \\
\text { Pre-Processing } \\
\text { Techniques (Histogram } \\
\text { Equalization and } \\
\text { Gaussian Filter) }\end{array}$ \\
\hline $\begin{array}{c}\text { Original MMU } \\
\text { database Images }\end{array}$ & 450 & 450 & 450 & 450 \\
\hline $\begin{array}{c}\text { The MMU database } \\
\text { images altered with } \\
\text { reducing contrast by } \\
\text { 40\%. }\end{array}$ & 450 & 398 & 426 & 287 \\
\hline $\begin{array}{c}\text { The MMU } \\
\text { database images } \\
\text { altered with }\end{array}$ & 450 & 317 & 374 & 204 \\
\hline
\end{tabular}

increasing contrast and brightness by $40 \%$.

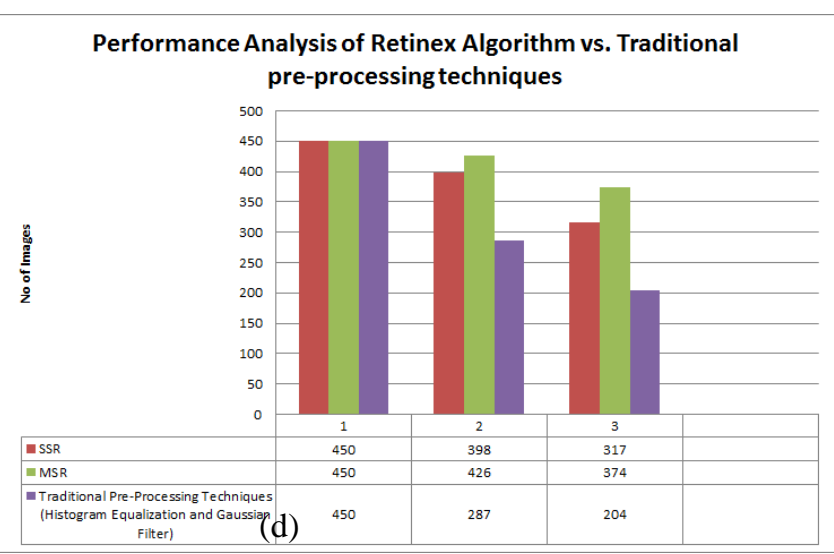

Fig 6: The performance analysis of Retinex algorithm vs. traditional pre-processing techniques

\section{Conclusion}

The proposed work implements Retinex algorithm for improving iris detection and recognition rate. In this work Single scale Retinex and Multi scale Retinex algorithm is implemented. The algorithm uses MMU iris database for evaluation. The results evaluated into three different cases: 1. Original MMU database Images. 2. The MMU database images altered with contrast by $40 \%$. 3. The MMU database images altered with contrast and brightness by $40 \%$. The results show our proposed work provide better results.

\section{References}

[1] Lenka R \& Khandual A, "A Study on Retinex Theory and Illumination Effects I", International Journal of Advanced Research in Computer Science and Software Engineering, Vol.6, No.1, (2016).

[2] Daugman J, "How iris recognition works", The essential guide to image processing, (2009), pp. 715-739.

[3] Daugman John G, "Biometric personal identification system based on iris analysis", U.S. Patent, (1994), pp. 291-560.

[4] Biswas R, Jia U \& Md Junayed H, "A New Approach of Iris Detection and Recognition", International Journal of Electrical and Computer Engineering (IJECE), Vol.7, No.5, (2017), pp.2530-2536.

[5] Varshney A, Asha R \& Vijander S, "Optimization of filter parameters for iris detection", 4th International Conference on Reliability, Infocom Technologies and Optimization (ICRITO) (Trends and Future Directions), (2015).

[6] Karthika R \& Latha P, "Study of Gabor wavelet for face recognition invariant to pose and orientation", Proceedings of the International Conference on Soft Computing Systems. Springer, New Delhi, (2016).

[7] Prathilothamai M \& Priyanka Sunil N, "De-duplication of passports using Aadhaar", IEEE International Conference on Computer Communication and Informatics, (2017). 